\title{
MoJiTalk: Generating Emotional Responses at Scale
}

\author{
Xianda Zhou \\ Dept. of Computer Science and Technology Department of Computer Science \\ Tsinghua University \\ Beijing, 100084 China \\ University of California, Santa Barbara \\ Santa Barbara, CA 93106 USA \\ zhou-xd13@mails.tsinghua.edu.cn williamecs.ucsb.edu
}

\begin{abstract}
Generating emotional language is a key step towards building empathetic natural language processing agents. However, a major challenge for this line of research is the lack of large-scale labeled training data, and previous studies are limited to only small sets of human annotated sentiment labels. Additionally, explicitly controlling the emotion and sentiment of generated text is also difficult. In this paper, we take a more radical approach: we exploit the idea of leveraging Twitter data that are naturally labeled with emojis.

We collect a large corpus of Twitter conversations that include emojis in the response and assume the emojis convey the underlying emotions of the sentence. We investigate several conditional variational autoencoders training on these conversations, which allow us to use emojis to control the emotion of the generated text. Experimentally, we show in our quantitative and qualitative analyses that the proposed models can successfully generate highquality abstractive conversation responses in accordance with designated emotions.
\end{abstract}

\section{Introduction}

A critical research problem for artificial intelligence is to design intelligent agents that can perceive and generate human emotions. In the past decade, there has been significant progress in sentiment analysis (Pang et al., 2002, 2008; Liu, 2012) and natural language understanding - e.g., classifying the sentiment of online reviews. To build empathetic conversational agents, machines must also have the ability to learn to generate emotional sentences.

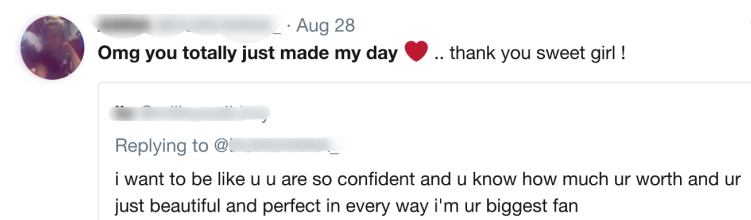

Figure 1: An example Twitter conversation with emoji in the response (top). We collected a large amount of these conversations, and trained a reinforced conditional variational autoencoder model to automatically generate abstractive emotional responses given any emoji.

One of the major challenges is the lack of largescale, manually labeled emotional text datasets. Due to the cost and complexity of manual annotation, most prior research studies primarily focus on small-sized labeled datasets (Pang et al., 2002; Maas et al., 2011; Socher et al., 2013), which are not ideal for training deep learning models with a large number of parameters.

In recent years, a handful of medium to large scale, emotional corpora in the area of emotion analysis (Go et al., 2016) and dialog ( $\mathrm{Li}$ et al., 2017b) are proposed. However, all of them are limited to a traditional, small set of labels, for example, "happiness," "sadness," "anger," etc. or simply binary "positive" and "negative." Such coarse-grained classification labels make it difficult to capture the nuances of human emotion.

To avoid the cost of human annotation, we propose the use of naturally-occurring emoji-rich Twitter data. We construct a dataset using Twitter conversations with emojis in the response. The fine-grained emojis chosen by the users in the response can be seen as the natural label for the emotion of the response.

We assume that the emotions and nuances of emojis are established through the extensive usage by Twitter users. If we can create agents that 
are able to imitate Twitter users' language style when using those emojis, we claim that, to some extent, we have captured those emotions. Using a large collection of Twitter conversations, we then trained a conditional generative model to automatically generate the emotional responses. Figure 1 shows an example.

To generate emotional responses in dialogs, another technical challenge is to control the target emotion labels. In contrast to existing work (Huang et al., 2017) that uses information retrieval to generate emotional responses, the research question we are pursuing in this paper, is to design novel techniques that can generate abstractive responses of any given arbitrary emotions, without having human annotators to label a huge amount of training data.

To control the target emotion of the response, we investigate several encoder-decoder generation models, including a standard attention-based SEQ2SEQ model as the base model, and a more sophisticated CVAE model (Kingma and Welling, 2013; Sohn et al., 2015), as VAE is recently found convenient in dialog generation (Zhao et al., 2017).

To explicitly improve emotion expression, we then experiment with several extensions to the CVAE model, including a hybrid objective with policy gradient. The performance in emotion expression is automatically evaluated by a separate sentence-to-emoji classifier (Felbo et al., 2017). Additionally, we conducted a human evaluation to assess the quality of the generated emotional text.

Results suggest that our method is capable of generating state-of-the-art emotional text at scale. Our main contributions are three-fold:

- We provide a publicly available, large-scale dataset of Twitter conversation-pairs naturally labeled with fine-grained emojis.

- We are the first to use naturally labeled emojis for conducting large-scale emotional response generation for dialog.

- We apply several state-of-the-art generative models to train an emotional response generation system, and analysis confirms that our models deliver strong performance.

In the next section, we outline related work on sentiment analysis and emoji on Twitter data, as well as neural generative models. Then, we will introduce our new emotional research dataset and formalize the task. Next, we will describe the neural models we applied for the task. Finally, we will show automatic evaluation and human evaluation results, and some generated examples. Experiment details can be found in supplementary materials.

\section{Related Work}

In natural language processing, sentiment analysis (Pang et al., 2002) is an area that involves designing algorithms for understanding emotional text. Our work is aligned with some recent studies on using emoji-rich Twitter data for sentiment classification. Eisner et al. (2016) proposes a method for training emoji embedding EMOJI2VEC, and combined with word2vec (Mikolov et al., 2013), they apply the embeddings for sentiment classification. DeepMoji (Felbo et al., 2017) is closely related to our study: It makes use of a large, naturally labeled Twitter emoji dataset, and train an attentive bi-directional long short-term memory network (Hochreiter and Schmidhuber, 1997) model for sentiment analysis. Instead of building a sentiment classifier, our work focuses on generating emotional responses, given the context and the target emoji.

Our work is also in line with the recent progress of the application of Variational Autoencoder (VAE) (Kingma and Welling, 2013) in dialog generation. VAE (Kingma and Welling, 2013) encodes data in a probability distribution, and then samples from the distribution to generate examples. However, the original frameworks do not support end-to-end generation. Conditional VAE (CVAE) (Sohn et al., 2015; Larsen et al., 2015) was proposed to incorporate conditioning option in the generative process. Recent research in dialog generation shows that language generated by VAE models enjoy significantly greater diversity than traditional SEQ2SEQ models (Zhao et al., 2017), which is a preferable property toward building a true-to-life dialog agents.

In dialog research, our work aligns with recent advances in sequence-to-sequence models (Sutskever et al., 2014) using long shortterm memory networks (Hochreiter and Schmidhuber, 1997). A slightly altered version of this model serves as our base model. Our modification enabled it to condition on single emojis. $\mathrm{Li}$ 


\begin{tabular}{|c|c|c|c|}
\hline$\Leftrightarrow 184,500$ & $=9,505$ & $\fallingdotseq 5,558$ & iㅣ 2,771 \\
\hline 6) $\quad 38,479$ & 9,455 & C5,114 & 2,532 \\
\hline 30,447 & $\Leftrightarrow 9,298$ & ㄷ. 5,026 & $\Rightarrow 2,332$ \\
\hline 25,018 & $\Leftrightarrow 8,385$ & $\underline{100} 4,738$ & $\Leftrightarrow 2,293$ \\
\hline 19,832 & 8,341 & 4,623 & 1,698 \\
\hline 16,934 & 8,293 & $\Leftrightarrow 4,531$ & 1,534 \\
\hline 17,009 & 8,144 & 4,287 & 1,403 \\
\hline 15,563 & 7,101 & $=4,205$ & ses 1,258 \\
\hline 15,046 & (6.) 6,939 & 64,066 & 1,091 \\
\hline 14,121 & ;) 6,769 & 3,973 & 698 \\
\hline 13,887 & 6,625 & (2) 3,841 & 627 \\
\hline 13,741 & 숑 6,558 & $\Leftrightarrow 3,863$ & 423 \\
\hline 13,147 & 6,374 & 3,236 & 250 \\
\hline 10,927 & $\therefore 6,031$ & $+3,072$ & 243 \\
\hline 10,104 & . 5,849 & 우 3,088 & 154 \\
\hline 9,546 & क5 5,624 & 장 2,969 & 尺ि \\
\hline
\end{tabular}

Table 1: All 64 emoji labels, and number of conversations labeled by each emoji.

et al. (2016) use a reinforcement learning algorithm to improve the vanilla sequence-to-sequence model for non-task-oriented dialog systems, but their reinforced and its follow-up adversarial models (Li et al., 2017a) also do not model emotions or conditional labels. Zhao et al. (2017) recently introduced conditional VAE for dialog modeling, but neither did they model emotions in the conversations, nor explore reinforcement learning to improve results. Given a dialog history, Xie et. al.'s work recommends suitable emojis for current conversation. Xie et. al. (2016)compress the dia$\log$ history to vector representation through a hierarchical RNN and then map it to a emoji by a classifier, while in our model, the representation for original tweet, combined with the emoji embedding, is used to generate a response.

\section{Dataset}

We start by describing our dataset and approaches to collecting and processing the data. Social media is a natural source of conversations, and people use emojis extensively within their posts. However, not all emojis are used to express emotion and frequency of emojis are unevenly distributed. Inspired by DeepMoji (Felbo et al., 2017), we use 64 common emojis as labels (see Table 1), and collect a large corpus of Twitter conversations con-
Before: @amy miss you soooo much!!!

After: miss you soo much!

Label:

Figure 2: An artificial example illustrating preprocess procedure and choice of emoji label. Note that emoji occurrences in responses are counted before the deduplication process.

taining those emojis. Note that emojis with the difference only in skin tone are considered the same emoji.

\subsection{Data Collection}

We crawled conversation pairs consisting of an original post and a response on Twitter from 12th to 14th of August, 2017. The response to a conversation must include at least one of the 64 emoji labels. Due to the limit of Twitter streaming API, tweets are filtered on the basis of words. In our case, a tweet can be reached only if at least one of the 64 emojis is used as a word, meaning it has to be a single character separated by blank space. However, this kind of tweets is arguably cleaner, as it is often the case that this emoji is used to wrap up the whole post and clusters of repeated emojis are less likely to appear in such tweets.

For both original tweets and responses, only English tweets without multimedia contents (such as URL, image or video) are allowed, since we assume that those contents are as important as the text itself for the machine to understand the conversation. If a tweet contains less than three alphabetical words, the conversation is not included in the dataset.

\subsection{Emoji Labeling}

Then we label responses with emojis. If there are multiple types of emoji in a response, we use the emoji with most occurrences inside the response. Among those emojis with same occurrences, we choose the least frequent one across the whole corpus, on the hypothesis that less frequent tokens better represent what the user wants to express. See Figure 2 for example. 


\subsection{Data Preprocessing}

During preprocessing, all mentions and hashtags are removed, and punctuation ${ }^{1}$ and emojis are separated if they are adjacent to words. Words with digits are all treated as the same special token.

In some cases, users use emojis and symbols in a cluster to express emotion extensively. To normalize the data, words with more than two repeated letters, symbol strings of more than one repeated punctuation symbols or emojis are shortened, for example, '!!!!' is shortened to '!', and 'yessss' to 'yess'. Note that we do not reduce duplicate letters completely and convert the word to the 'correct' spelling ('yes' in the example) since the length of repeated letters represents the intensity of emotion. By distinguishing 'yess' from 'yes', the emotional intensity is partially preserved in our dataset.

Then all symbols, emojis, and words are tokenized. Finally, we build a vocabulary of size $20 \mathrm{~K}$ according to token frequency. Any tokens outside the vocabulary are replaced by the same special token.

We randomly split the corpus into 596,959 132,600/32,600 conversation pairs for train /validation/test set ${ }^{2}$. Distribution of emoji labels within the corpus is presented in Table 1.

\section{Generative Models}

In this work, our goal is to generate emotional responses to tweets with the emotion specified by an emoji label. We assembled several generative models and trained them on our dataset.

\subsection{Base: Attention-Based Sequence-to-Sequence Model}

Traditional studies use deep recurrent architecture and encoder-decoder models to generate conversation responses, mapping original texts to target responses. Here we use a sequence-to-sequence (SEQ2SEQ) model (Sutskever et al., 2014) with global attention mechanism (Luong et al., 2015) as our base model (See Figure 3).

We use randomly initialized embedding vectors to represent each word. To specifically model the

\footnotetext{
${ }^{1}$ Emoticons (e.g. ':)', '(-:') are made of mostly punctuation marks. They are not examined in this paper. Common emoticons are treated as words during preprocessing.

${ }^{2} \mathrm{We}$ will release the dataset with all tweets in its original form before preprocessing. To comply with Twitter's policy, we will include the tweet IDs in our release, and provide a script for downloading the tweets using the official API. No information of the tweet posters is collected.
}

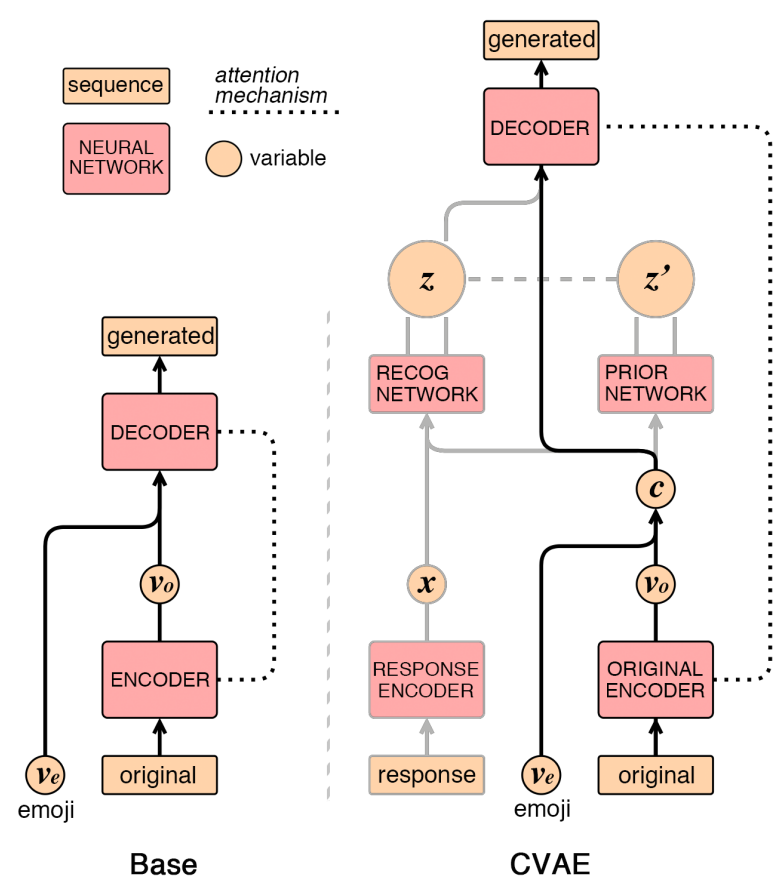

Figure 3: From bottom to top is a forward pass of data during training. Left: the base model encodes the original tweets in $v_{o}$, and generates responses by decoding from the concatenation of $v_{o}$ and the embedded emoji, $v_{e}$. Right: In the CVAE model, all additional components (outlined in gray) can be added incrementally to the base model. A separate encoder encodes the responses in $x$. Recognition network inputs $x$ and produces the latent variable $z$ by reparameterization trick. During training, The latent variable $z$ is concatenated with $v_{o}$ and $v_{e}$ and fed to the decoder.

emotion, we compute the embedding vector of the emoji label the same way as word embeddings. The emoji embedding is further reduced to smaller size vector $v_{e}$ through a dense layer. We pass the embeddings of original tweets through a bidirectional RNN encoder of GRU cells (Schuster and Paliwal, 1997; Chung et al., 2014). The encoder outputs a vector $v_{o}$ that represents the original tweet. Then $v_{o}$ and $v_{e}$ are concatenated and fed to a 1-layer RNN decoder of GRU cells. A response is then generated from the decoder.

\subsection{Conditional Variational Autoencoder (CVAE)}

Having similar encoder-decoder structures, SEQ2SEQ can be easily extended to a Conditional Variational Autoencoder (CVAE) (Sohn et al., 2015). Figure 3 illustrates the model: response encoder, recognition network, and prior network 
are added on top of the SEQ2SEQ model. Response encoder has the same structure to original tweet encoder, but it has separate parameters. We use embeddings to represent Twitter responses and pass them through response encoder.

Mathematically, CVAE is trained by maximizing a variational lower bound on the conditional likelihood of $x$ given $c$, according to:

$$
p(x \mid c)=\int p(x \mid z, c) p(z \mid c) d z
$$

$z, c$ and $x$ are random variables. $z$ is the latent variable. In our case, the condition $c=$ $\left[v_{o} ; v_{e}\right]$, target $x$ represents the response. Decoder is used to approximate $p(x \mid z, c)$, denoted as $p_{D}(x \mid z, c)$. Prior network is introduced to approximate $p(z \mid c)$, denoted as $p_{P}(z \mid c)$. Recognition network $q_{R}(z \mid x, c)$ is introduced to approximate true posterior $p(z \mid x, c)$ and will be absent during generation phase. By assuming that the latent variable has a multivariate Gaussian distribution with a diagonal covariance matrix, the lower bound to $\log p(x \mid c)$ can then be written by:

$$
\begin{array}{r}
-\mathcal{L}\left(\theta_{D}, \theta_{P}, \theta_{R} ; x, c\right)=\operatorname{KL}\left(q_{R}(z \mid x, c) \| p_{P}(z \mid c)\right) \\
-\mathbb{E}_{q_{R}(z \mid x, c)}\left(\log p_{D}(x \mid z, c)\right)
\end{array}
$$

$\theta_{D}, \theta_{P}, \theta_{R}$ are parameters of those networks.

In recognition/prior network, we first pass the variables through an MLP to get the mean and log variance of $z$ 's distribution. Then we run a reparameterization trick (Kingma and Welling, 2013) to sample latent variables. During training, $z$ by the recognition network is passed to the decoder and trained to approximate $z^{\prime}$ by the prior network. While during testing, the target response is absent, and $z^{\prime}$ by the prior network is passed to the decoder.

Our CVAE inherits the same attention mechanism from the base model connecting the original tweet encoder to the decoder, which makes our model deviate from previous works of CVAE on text data. Based on the attention memory as well as $c$ and $z$, a response is finally generated from the decoder.

When handling text data, the VAE models that apply recurrent neural networks as the structure of their encoders/decoders may first learn to ignore the latent variable, and explain the data with the more easily optimized decoder. The latent variables lose its functionality, and the VAE deteriorates to a plain SEQ2SEQ model mathematically (Bowman et al., 2015). Some previous methods effectively alleviate this problem. Such methods are also important to keep a balance between the two items of the loss, namely KL loss and reconstruction loss. We use techniques of KL annealing, early stopping (Bowman et al., 2015) and bag-of-word loss (Zhao et al., 2017) in our models. The general loss with bag-of-word loss (see supplementary materials for details) is rewritten as:

$$
\mathcal{L}^{\prime}=\mathcal{L}+\mathcal{L}_{\text {bow }}
$$

\subsection{Reinforced CVAE}

In order to further control the emotion of our generation more explicitly, we combine policy gradient techniques on top of the CVAE above and proposed Reinforced CVAE model for our task. We first train an emoji classifier on our dataset separately and fix its parameters thereafter. The classifier is used to produce reward for the policy training. It is a skip connected model of Bidirectional GRU-RNN layers (Felbo et al., 2017).

During the policy training, we first get the generated response $x^{\prime}$ by passing $x$ and $c$ through the CVAE, then feeding generation $x^{\prime}$ to classifier and get the probability of the emoji label as reward $R$. Let $\theta$ be parameters of our network, REINFORCE algorithm (Williams, 1992) is used to maximize the expected reward of generated responses:

$$
\mathcal{J}(\theta)=\mathbb{E}_{p(x \mid c)}\left(R_{\theta}(x, c)\right)
$$

The gradient of Equation 4 is approximated using the likelihood ratio trick (Glynn, 1990; Williams, 1992):

$$
\nabla \mathcal{J}(\theta)=(R-r) \nabla \sum_{t}^{|x|} \log p\left(x_{t} \mid c, x_{1: t-1}\right)
$$

$r$ is the baseline value to keep estimate unbiased and reduce its variance. In our case, we directly pass $x$ through emoji classifier and compute the probability of the emoji label as $r$. The model then encourages response generation that has $R>r$.

As REINFORCE objective is unrelated to response generation, it may make the generation model quickly deteriorate to some generic responses. To stabilize the training process, we propose two straightforward techniques to constrain the policy training: 
1. Adjust rewards according to the position of the emoji label when all labels are ranked from high to low in order of the probability given by the emoji classifier. When the probability of the emoji label is of high rank among all possible emojis, we assume that the model has succeeded in emotion expression, thus there is no need to adjust parameters toward higher probability in this response. Modified policy gradient is written as:

$$
\nabla \mathcal{J}^{\prime}(\theta)=\alpha(R-r) \nabla \sum_{t}^{|x|} \log p\left(x_{t} \mid c, x_{1: t-1}\right)
$$

where $\alpha \in[0,1]$ is a variant coefficient. The higher $R$ ranks in all types of emoji label, the closer $\alpha$ is to 0 .

2. Train Reinforced CVAE by a hybrid objective of REINFORCE and variational lower bound objective, learning towards both emotion accuracy and response appropriateness:

$$
\min _{\theta} \mathcal{L}^{\prime \prime}=\mathcal{L}^{\prime}-\lambda \mathcal{J}^{\prime}
$$

$\lambda$ is a balancing coefficient, which is set to 1 in our experiments.

The algorithm outlining the training process of Reinforced CVAE can be found in the supplementary materials.

\section{Experimental Results and Analyses}

We conducted several experiments to finalize the hyper-parameters of our models (Table 2). During training, fully converged base SEQ2SEQ model is used to initialize its counterparts in CVAE models. Pretraining is vital to the success of our models since it is essentially hard for them to learn a latent variable space from total randomness. For more details, please refer to the supplementary materials.

In this section, we first report and analyze the general results of our models, including perplexity, loss and emotion accuracy. Then we take a closer look at the generation quality as well as our models' capability of expressing emotion.

\subsection{General}

To generally evaluate the performance of our models, we use generation perplexity and top- $1 /$ top- 5

\begin{tabular}{l|ccc}
\hline & \multicolumn{3}{|c}{ Emoji Accuracy } \\
\cline { 2 - 4 } Model & Perplexity & Top1 & Top5 \\
\hline & 127.0 & $34.2 \%$ & $57.6 \%$ \\
\hline Base & 37.1 & $40.7 \%$ & $75.3 \%$ \\
CVAE & 38.1 & $42.2 \%$ & $76.9 \%$ \\
Reinforced CVAE & \multicolumn{3}{|c}{ Test } \\
\hline & 130.6 & $33.9 \%$ & $58.1 \%$ \\
\hline Base & 36.9 & $41.4 \%$ & $75.1 \%$ \\
CVAE & 38.3 & $42.1 \%$ & $77.3 \%$ \\
Reinforced CVAE & & & \\
\hline
\end{tabular}

Table 2: Generation perplexity and emoji accuracy of the three models.

emoji accuracy on the test set. Perplexity indicates how much difficulty the model is having when generating responses. We also use top-5 emoji accuracy, since the meaning of different emojis may overlap with only a subtle difference. The machine may learn that similarity and give multiple possible labels as the answer.

Note that we use the same emoji classifier for evaluation. Its accuracy (see supplementary materials) may not seem perfect, but it is the stateof-the-art emoji classifier given so many classes. Also, it's reasonable to use the same classifier in training for automated evaluation, as is in $(\mathrm{Hu}$ et al., 2017). We can obtain meaningful results as long as the classifier is able to capture the semantic relationship between emojis (Felbo et al., 2017).

As is shown in Table 2, CVAE significantly reduces the perplexity and increases the emoji accuracy over base model. Reinforced CVAE also adds to the emoji accuracy at the cost of a slight increase in perplexity. These results confirm that proposed methods are effective toward the generation of emotional responses.

When converged, the KL loss is $27.0 / 25.5$ for the CVAE/Reinforced CVAE respectively, and reconstruction loss 42.2/40.0. The models achieved a balance between the two items of loss, confirming that they have successfully learned a meaningful latent variable.

\subsection{Generation Diversity}

SEQ2SEQ generates in a monotonous way, as several generic responses occur repeatedly, while the generation of CVAE models is of much more diversity. To showcase this disparity, we calculated the type-token ratios of unigrams/bigrams/trigrams in generated responses as 

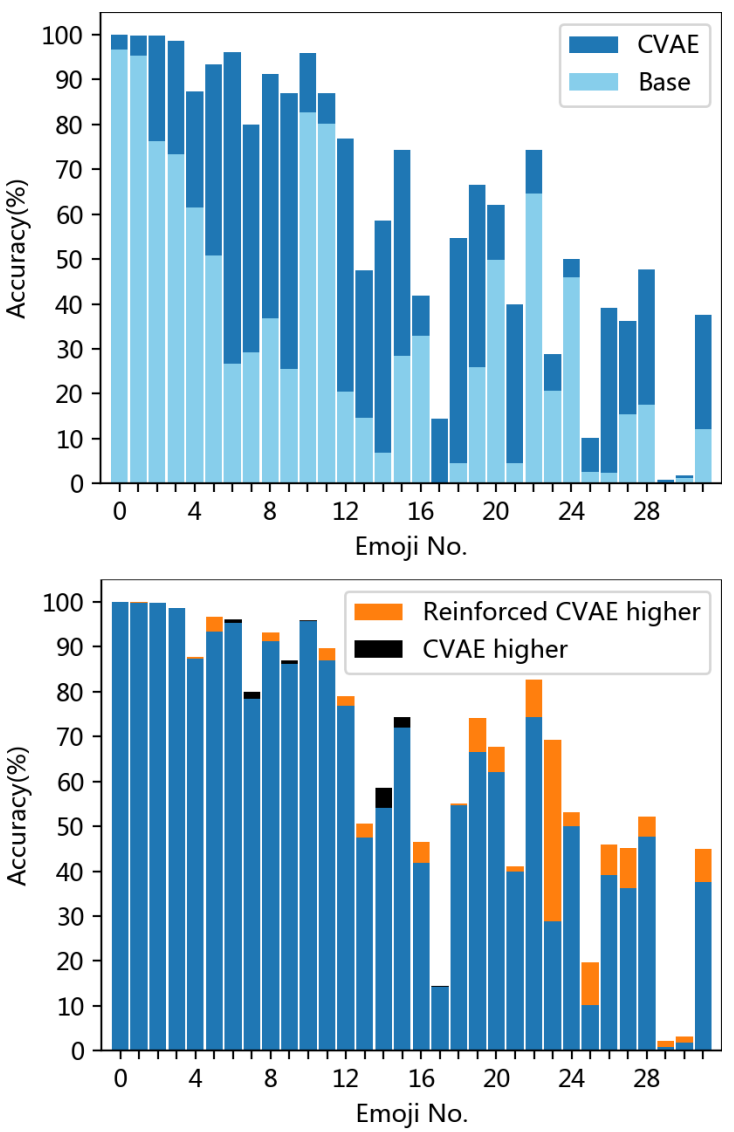

Figure 4: Top5 emoji accuracy of the first 32 emoji labels. Each bar represents an emoji and its length represents how many of all responses to the original tweets are top5 accurate. Different colors represent different models. Emojis are numbered in the order of frequencies in the dataset. No.0 is $\hat{\theta}$, for instance, No.1 sol and so on.

Top: CVAE v. Base.

Bottom: Reinforced CVAE v. CVAE. If Reinforced CVAE scores higher, the margin is marked in orange. If lower, in black.

the diversity score.

As shown in Table 3, results show that CVAE models beat the base models by a large margin. Diversity scores of Reinforced CVAE are reasonably compromised since it's generating more emotional responses.

\subsection{Controllability of Emotions}

There are potentially multiple types of emotion in reaction to an utterance. Our work makes it possible to generate a response to an arbitrary emotion by conditioning the generation on a specific type of emoji. In this section, we generate one response in reply to each original tweet in the dataset and condition on each emoji of the selected 64 emo-

\begin{tabular}{l|ccc}
\hline Model & Unigram & Bi- & Tri- \\
\hline \hline Base & 0.0061 & 0.0199 & 0.0362 \\
CVAE & 0.0191 & 0.131 & 0.365 \\
Reinforced CVAE & 0.0160 & 0.118 & 0.337 \\
\hline \hline Target responses & 0.0353 & 0.370 & 0.757 \\
\hline
\end{tabular}

Table 3: Type-token ratios of the generation by the three models. Scores of tokenized humangenerated target responses are given for reference.

\begin{tabular}{l|l|ccc}
\hline Setting & Model v. Base & Win & Lose & Tie \\
\hline \hline reply & CVAE & $42.4 \%$ & $43.0 \%$ & $14.6 \%$ \\
reply & Reinforced CVAE & $40.6 \%$ & $39.6 \%$ & $19.8 \%$ \\
\hline emoji & CVAE & $48.4 \%$ & $26.2 \%$ & $25.4 \%$ \\
emoji & Reinforced CVAE & $50.0 \%$ & $19.6 \%$ & $30.4 \%$ \\
\hline
\end{tabular}

Table 4: Results of human evaluation. Tests are conducted pairwise between CVAE models and the base model.

jis. We may have recorded some original tweets with different replies in the dataset, but an original tweet only need to be used once for each emoji, so we eliminate duplicate original tweets in the dataset. There are 30,299 unique original tweets in the test set.

Figure 4 shows the top- 5 accuracy of each type of the first 32 emoji labels when the models generates responses from the test set conditioning on the same emoji. The results show that CVAE models increase the accuracy over every type of emoji label. Reinforced CVAE model sees a bigger increase on the less common emojis, confirming the effect of the emoji-specified policy training.

\subsection{Human Evaluation}

We employed crowdsourced judges to evaluate a random sample of 100 items (Table 4), each being assigned to 5 judges on the Amazon Mechanical Turk. We present judges original tweets and generated responses. In the first setting of human evaluation, judges are asked to decide which one of the two generated responses better reply the original tweet. In the second setting, the emoji label is presented with the item discription, and judges are asked to pick one of the two generated responses that they decide better fits this emoji. (These two settings of evaluation are conducted separately so that it will not affect judges' verdicts.) Order of two generated responses under one item is permuted. Ties are permitted for an- 


\begin{tabular}{|c|c|c|c|}
\hline Content & \multicolumn{3}{|c|}{$\begin{array}{l}\text { sorry guys, was gunna stream tonight but i 'm still feeling like crap and my voice disappeared. } \\
\text { i will make it up to you }\end{array}$} \\
\hline Target Emotion & $\frac{\sigma}{3}=$ & als & $\because 0$ \\
\hline Base & $\begin{array}{l}\text { i 'm sorry you 're going to be } \\
\text { missed it }\end{array}$ & i 'm sorry for your loss & $\begin{array}{l}\text { i 'm sorry you 're going to be } \\
\text { able to get it }\end{array}$ \\
\hline CVAE & hope you are okay hun! & hi jason, i'll be praying for you & im sorry u better suck u off \\
\hline $\begin{array}{l}\text { Reinforced } \\
\text { CVAE }\end{array}$ & hope you 're feeling it & $\begin{array}{l}\text { hope you had a speedy recovery } \\
\text { man! hope you feel better soon } \\
\text {, please get well soon }\end{array}$ & $\begin{array}{l}\text { dude } \mathrm{i} \text { 'm so sorry for that } \mathrm{i} \\
\text { wanna hear it and } \mathrm{i} \text { 'm sorry } \mathrm{i} \\
\text { can 't go to canada with you but } \\
\text { i wanna be away from canada }\end{array}$ \\
\hline Content & \multicolumn{3}{|l|}{ add me in there my bro } \\
\hline Target Emotion & 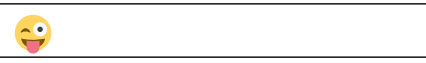 & 5 & ( \\
\hline Base & i 'm not sure you 'll be there & i'm here for you & i 'm not ready for you \\
\hline CVAE & $\begin{array}{l}\text { you know, you need to tell me } \\
\text { in your hometown! }\end{array}$ & $\begin{array}{l}\text { you will be fine bro, } \mathrm{i} \text { 'll be in } \\
\text { the gym for you }\end{array}$ & i can't wait $\cong$ \\
\hline $\begin{array}{l}\text { Reinforced } \\
\text { CVAE }\end{array}$ & $\begin{array}{l}\text { you might have to get me hip } \\
\text { hop off. }\end{array}$ & $\begin{array}{l}\text { good luck bro ! this is about to } \\
\text { be healthy }\end{array}$ & $\begin{array}{l}\text { i 'm still undecided and i 'm still } \\
\text { waiting }\end{array}$ \\
\hline Content & \multicolumn{3}{|c|}{ don 't tell me match of the day is delayed because of this shit } \\
\hline Target Emotion & $\Leftrightarrow$ & 臬 & $\Leftrightarrow$ \\
\hline Base & i 'm not even a fan of the game & $\begin{array}{l}\mathrm{i} \text { 'm not sure if you ever have } \\
\text { any chance to talk to someone } \\
\text { else }\end{array}$ & i 'm sorry i'm not doubting you \\
\hline CVAE & $\begin{array}{l}\text { you can 't do it bc you 're in my } \\
\text { mentions }\end{array}$ & see now a good point & hiya, unfortunately, it's not \\
\hline $\begin{array}{l}\text { Reinforced } \\
\text { CVAE }\end{array}$ & $\begin{array}{l}\text { oh my god i 'm saying this as } \\
\text { long as i remember my twitter }\end{array}$ & $\begin{array}{l}\text { fab mate, you 'll enjoy the } \\
\text { game and you 'll get a win }\end{array}$ & it 's the worst \\
\hline Content & \multicolumn{3}{|l|}{$\mathrm{g}$ i needed that laugh lmfaoo } \\
\hline Target Emotion & 0 & $\Leftrightarrow$ & $\because$ \\
\hline Base & i'm glad you enjoyed it & i'm not gonna lie & i 'm sorry i 'm not laughing \\
\hline CVAE & good! have a good time & i don 't plan on that & me too . but it's a lot of me. \\
\hline $\begin{array}{l}\text { Reinforced } \\
\text { CVAE }\end{array}$ & $\begin{array}{l}\text { thank you for your tweet, you } \\
\text { didn't know how much i guess }\end{array}$ & $\begin{array}{l}\text { that 's a bad idea, u gotta hit me } \\
\text { up on my phone }\end{array}$ & $\begin{array}{l}\text { i feel bad at this and i hope you } \\
\text { can make a joke }\end{array}$ \\
\hline
\end{tabular}

Table 5: Some examples from our generated emotional responses. Context is the original tweet, and target emotion is specified by the emoji. Following are the responses generated by each of the three models based on the context and the target emotion.

swers. We batch five items as one assignment and insert an item with two identical outputs as the sanity check. Anyone who failed to choose "tie" for that item is considered as a careless judge and is therefore rejected from our test.

We then conducted a simplified Turing test. Each item we present judges an original tweet, its reply by a human, and its response generated from Reinforced CVAE model. We ask judges to decide which of the two given responses is written by a human. Other parts of the setting are similar to above-mentioned tests. It turned out $18 \%$ of the test subjects mistakenly chose machine-generated responses as human written, and $27 \%$ stated that they were not able to distinguish between the two responses.

In regard of the inter-rater agreement, there are four cases. The ideal situation is that all five judges choose the same answer for a item, and in the worst-case scenario, at most two judges choose the same answer. In light of this, we have counted that $32 \% / 33 \% / 31 \% / 5 \%$ of all items have 5/4/3/2 judges in agreement, showing that our experiment has a reasonably reliable inter-rater agreement.

\subsection{Case Study}

We sampled some generated responses from all three models, and list them in Figure 5. Given 
an original tweet, we would like to generate responses with three different target emotions.

SEQ2SEQ only chooses to generate most frequent expressions, forming a predictable pattern for its generation (See how every sampled response by the base model starts with "I'm"). On the contrary, generation from the CVAE model is diverse, which is in line with previous quantitative analysis. However, the generated responses are sometimes too diversified and unlikely to reply to the original tweet.

Reinforced CVAE somtetimes tends to generate a lengthy response by stacking up sentences (See the responses to the first tweet when conditioning on the 'folded hands' emoji and the 'sad face' emoji). It learns to break the length limit of sequence generation during hybrid training, since the variational lower bound objective is competing with REINFORCE objective. The situation would be more serious is $\lambda$ in Equation 7 is set higher. However, this phenomenon does not impair the fluency of generated sentences, as can be seen in Figure 5.

\section{Conclusion and Future Work}

In this paper, we investigate the possibility of using naturally annotated emoji-rich Twitter data for emotional response generation. More specifically, we collected more than half a million Twitter conversations with emoji in the response and assumed that the fine-grained emoji label chosen by the user expresses the emotion of the tweet. We applied several state-of-the-art neural models to learn a generation system that is capable of giving a response with an arbitrarily designated emotion. We performed automatic and human evaluations to understand the quality of generated responses. We trained a large scale emoji classifier and ran the classifier on the generated responses to evaluate the emotion accuracy of the generated response. We performed an Amazon Mechanical Turk experiment, by which we compared our models with a baseline sequence-to-sequence model on metrics of relevance and emotion. Experimentally, it is shown that our model is capable of generating high-quality emotional responses, without the need of laborious human annotations. Our work is a crucial step towards building intelligent dialog agents. We are also looking forward to transferring the idea of naturally-labeled emojis to task-oriented dialog and multi-turn dialog generation problems. Due to the nature of social media text, some emotions, such as fear and disgust, are underrepresented in the dataset, and the distribution of emojis is unbalanced to some extent. We will keep accumulating data and increase the ratio of underrepresented emojis, and advance toward more sophisticated abstractive generation methods. 


\section{References}

Samuel R. Bowman, Luke Vilnis, Oriol Vinyals, Andrew M. Dai, Rafal Józefowicz, and Samy Bengio. 2015. Generating sentences from a continuous space. CONLL.

Junyoung Chung, Çaglar Gülçehre, KyungHyun Cho, and Yoshua Bengio. 2014. Empirical evaluation of gated recurrent neural networks on sequence modeling. NIPS 2014 Deep Learning and Representation Learning Workshop.

Ben Eisner, Tim Rocktäschel, Isabelle Augenstein, Matko Bošnjak, and Sebastian Riedel. 2016. emoji2vec: Learning emoji representations from their description. SocialNLP at EMNLP.

Bjarke Felbo, Alan Mislove, Anders Søgaard, Iyad Rahwan, and Sune Lehmann. 2017. Using millions of emoji occurrences to learn any-domain representations for detecting sentiment, emotion and sarcasm. EMNLP.

Peter W Glynn. 1990. Likelihood ratio gradient estimation for stochastic systems. Communications of the ACM, 33(10):75-84.

Alec Go, Richa Bhayani, and Lei Huang. 2016. Sentiment140. http://help. sentiment 140 . $\mathrm{com} /$.

Sepp Hochreiter and Jürgen Schmidhuber. 1997. Long short-term memory. Neural computation, 9(8):1735-1780.

Zhiting $\mathrm{Hu}$, Zichao Yang, Xiaodan Liang, Ruslan Salakhutdinov, and Eric P Xing. 2017. Toward controlled generation of text. In International Conference on Machine Learning, pages 1587-1596.

Chieh-Yang Huang, Tristan Labetoulle, Ting-Hao Kenneth Huang, Yi-Pei Chen, Hung-Chen Chen, Vallari Srivastava, and Lun-Wei Ku. 2017. Moodswipe: A soft keyboard that suggests messages based on userspecified emotions. EMNLP Demo.

Diederik P Kingma and Max Welling. 2013. Autoencoding variational bayes. ICLR.

Anders Boesen Lindbo Larsen, Søren Kaae Sønderby, Hugo Larochelle, and Ole Winther. 2015. Autoencoding beyond pixels using a learned similarity metric. $I C M L$.

Jiwei Li, Will Monroe, Alan Ritter, and Dan Jurafsky. 2016. Deep reinforcement learning for dialogue generation. EMNLP.

Jiwei Li, Will Monroe, Tianlin Shi, Alan Ritter, and Dan Jurafsky. 2017a. Adversarial learning for neural dialogue generation. EMNLP.

Yanran Li, Hui Su, Xiaoyu Shen, Wenjie Li, Ziqiang Cao, and Shuzi Niu. 2017b. Dailydialog: A manually labelled multi-turn dialogue dataset. IJCNLP.
Bing Liu. 2012. Sentiment analysis and opinion mining. Synthesis lectures on human language technologies, 5(1):1-167.

Minh-Thang Luong, Hieu Pham, and Christopher D Manning. 2015. Effective approaches to attentionbased neural machine translation. EMNLP.

Andrew L Maas, Raymond E Daly, Peter T Pham, Dan Huang, Andrew Y Ng, and Christopher Potts. 2011. Learning word vectors for sentiment analysis. In Proceedings of the 49th Annual Meeting of the Association for Computational Linguistics: Human Language Technologies-Volume 1, pages 142-150. Association for Computational Linguistics.

Tomas Mikolov, Kai Chen, Greg Corrado, and Jeffrey Dean. 2013. Efficient estimation of word representations in vector space. ICLR.

Bo Pang, Lillian Lee, and Shivakumar Vaithyanathan. 2002. Thumbs up?: sentiment classification using machine learning techniques. In Proceedings of the ACL-02 conference on Empirical methods in natural language processing-Volume 10, pages 79-86. Association for Computational Linguistics.

Bo Pang, Lillian Lee, et al. 2008. Opinion mining and sentiment analysis. Foundations and Trends $\mathrm{R}$ in Information Retrieval, 2(1-2):1-135.

Mike Schuster and Kuldip K Paliwal. 1997. Bidirectional recurrent neural networks. IEEE Transactions on Signal Processing, 45(11):2673-2681.

Richard Socher, Alex Perelygin, Jean Wu, Jason Chuang, Christopher D Manning, Andrew Ng, and Christopher Potts. 2013. Recursive deep models for semantic compositionality over a sentiment treebank. In Proceedings of the 2013 conference on empirical methods in natural language processing, pages 1631-1642.

Kihyuk Sohn, Honglak Lee, and Xinchen Yan. 2015. Learning structured output representation using deep conditional generative models. In Advances in Neural Information Processing Systems, pages 3483-3491.

Ilya Sutskever, Oriol Vinyals, and Quoc V Le. 2014. Sequence to sequence learning with neural networks. In Advances in neural information processing systems, pages 3104-3112.

Ronald J Williams. 1992. Simple statistical gradientfollowing algorithms for connectionist reinforcement learning. Machine learning, 8(3-4):229-256.

Ruobing Xie, Zhiyuan Liu, Rui Yan, and Maosong Sun. 2016. Neural emoji recommendation in dialogue systems. arXiv preprint arXiv:1612.04609.

Tiancheng Zhao, Ran Zhao, and Maxine Eskenazi. 2017. Learning discourse-level diversity for neural dialog models using conditional variational autoencoders. ACL. 